\section{SECTION 13}

Laboratory techniques in molecular genetics

\subsection{2}

\section{Development of mini gene constructs of the} adenomatous polyposis coli gene

West, Sarah 1; Hamzehloei, T 2; Buddles, M 2; Curtis, A 1

' Northern Genetics Service, 19/20 Claremont Place, Newcastle upon Tyne, NE2 4AA; 2 Molecular Genetics Unit, Department of Human Genetics, Ridley Building, University of Newcatle upon Tyne, NE1 TRU

A simple, PCR based, procedure has been developed called exon linking for the production of partial constructs of the adenomatous polyposis coli coding sequence which allows the APC CDNA to be manipulated into a form which includes or excludes any part of the coding sequence. Exon linking is applicable to any gene for which the intron:exon boundaries are fully characterised. For example it is possible to generate constructs which include particular chosen mutations, or to select those parts of the gene which are regarded as functionally more significant than other parts and specifically incorporate these into mini versions of the gene. The procedure has three significant features: 1) Regions of the transcript are linked together without a prerequisite of being contiguous in the full length mRNA. 2) Specific exons can be included or excluded from the construct as required, in contrast to other mini genes which have been developed where naturally occurring, internally deleted, mRNA species have provided the source of CDNA. 3) Peripheral blood lymphocytes can be used as the tissue source since the procedure requires genomic DNA. There is no necessity for the preparation of CDNA from a tissue which expresses the sequence at high levels.

\subsection{3}

\section{Experience of a regional molecular genetics laboratory} in diagnosing HMSN1a and HNPP using a collection of polymorphic markers

Williams, Maggie 1; Tyfield, LA 1; Lunt, PW 2; Turnpenny, PD ${ }^{3}$

- Molecular Genetics Unit, The Lewis Laboratories, Southmead Hospital, Bristol; 2 Clinical Genetics, Institute of Child Health, Bristol; ${ }^{3}$ Clinical Genetics Service, Royal Devon \& Exeter Hospital, Exeter

HMSN1a and HNPP are autosomal dominant neuropathies associated with a duplication or deletion respectively of a $1.5 \mathrm{Mb}$ region at $17 \mathrm{p} 11.2$. Fifty-three patients from 40 families were tested for abnormalities at $17 \mathrm{p} 11.2$ using the STR and RFLP markers mapping to the $1.5 \mathrm{Mb}$ region: mfd41(D17S261), m11GT(D17S122), 191xh12(D17S921); 409R3a(D17S122), 412R3Heb, 412R3Hec(D17S125), 401He(D17S61). A diagnosis of HMSN1a was confirmed in 20 patients who had 3 alleles or dosage with two independent markers recorded by eye. Two patients, homozygous for all 7 markers, are believed to have HNPP. A report was issued in $98 \%$ of cases. Dosage detection using PCR is reliable if heterozygote alleles are $>2 \mathrm{bp}$ apart. Using this criterion the percentage reportable results generated by each marker was calculated. The most informative markers for a laboratory without an automated detection system are $191 \times \mathrm{xh} 12,409 \mathrm{R} 3 \mathrm{a}$, and $\mathrm{m} 11 \mathrm{GT}$, with $61 \%, 58 \%$ and $55 \%$ reportable results respectively. mfd41 with a heterozygosity of $40 \%$ was only reportable in $14 \%$ of cases. One patient was positive for the duplication at D17S122 and negative at D17S921. A rare polymorphism (allele $4.7 \mathrm{~kb}$, freq. $0.01 \%$ ) was detected with $401 \mathrm{He}$. A 3 yr old patient with cytogenetic duplication of 17 p11.2 was shown not to have HMSN1a by molecular testing.

\subsection{4}

Detection of dystrophin rearrangements by pulsed field gel electrophoresis (PFGE) in male Duchenne and Becker muscular dystrophy (DMD,BMD) where no deletion has previously been identified

Cockburn, David'; Clouston, P1; Hopkin, J2; Seller, A

1 Oxford Medical Genetics Laboratory, The Churchill, Oxford Radcliffe Hospital, Oxford OX3 7LJ; 2 Department of Clinical Genetics, The Churchill, Oxford Radcliffe Hospital, Oxford OX3 7LJ

The majority of male DMD/BMD patients are deleted for more than one dystrophin exon and these deletions are readily detected by multiplex PCR or Southern analysis using a selection of CDNA probes. Where these techniques did not identify any abnormality we have examined patients by PFGE. 37 DMD patients, including young boys where the clinical course is consistent with DMD, 15 patients with clinical evidence of BMD and 29 patients where BMD could not be excluded were examined. Among DMD patients, mutations were identified in 9/37 (24\%) comprising 6 duplications and 3 deletions ( 1 mistyped by multiplex PCR). Mutations were identified in 6/15 BMD patients (40\%) comprising 4 duplications and 2 deletions. No mutations were detected among the 29 patients where BMD was only a suspicion at time of referral. We conclude that duplications are detected at the expected frequency in DMD $(17 \%$ of undeleted patients). A significant number of deletions are detected which are missed by multiplex PCR and CDNA screens in DMD and BMD. In BMD where certainty of diagnosis is strong, PFGE detects a high frequency of further mutations, while if the diagnosis is only a suspicion results suggest most do not have a dystrophinopathy.

\subsection{5}

Cost analysis of dystrophin point mutation detection by reverse transcription - polymerase chain reaction

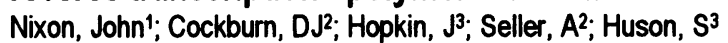

'University Department of Clinical Neurology, Radcliffe Infirmary, Oxford;20xford Medical Genetics Laboratories, Churchill Hospital, Oxford OX3 7LJ; ${ }^{3}$ Department of Clinical Genetics, Churchill Hospital, Oxford OX3 7LJ

The techniques routinely used at our laboratory for mutation detection in Duchenne muscular dystrophy (DMD) are multiplex PCR and pulsed field gel electrophoresis. Approximately $30 \%$ of DMD cases are due to point mutations, which cannot be detected by these methods. We have assessed reverse transcription - polymerase chain reaction (RT-PCR) and protein truncation test (PTT) for point mutation detection in affected males and obligate carrier (OC) females. Mutations were detected in 6 out of 12 affected males (4 of which still in progress). 11 females have had carrier tests, giving precise carrier risks in all except 2 mothers whose risks fell to germline mosaicism level. Four more females who were at significant risk consequently do not require testing. No mutations were detected in $5 \mathrm{OC}$ females, results from 2 known carriers of point mutations indicate that RTPCRIPTT is unlikely to be feasible for point mutation detection in OC females. Cost per family analysed ranges from $£ 650$ to $£ 1050$, showing that point mutation detection is more expensive than linkage analysis, but can give precise carrier risks and antenatal diagnosis where linkage analysis produces intermediate risks. We believe that RT-PCR/PTT should be made available as part of the routine DMD diagnostic service. 


\subsection{6}

Identification of an individual mosaic for Turner syndrome and a carrier of the FMR-1 expansion mutation.

Sharif, Abid 1; Pindar, L 2; Cooper, $C^{3}$; Ritchie, S J 4; McGhee, E 4; Cross, $\mathrm{S} \mathrm{S}^{1}$

Department of 1 Molecular Genetics, 3 Cytogenetics 'Clinical Genetics, Nottingham City Hospital, Nottingham NG5 1PB; 2 Department of Cytogenetics, Norfolk and Norwich Hospital, Brunswick Road, Norwich NR1 3SR

Patient ' $P$ ' with a family history of fragile $X$ and of normal intelligence was referred for fragile $X$ carrier testing. On an EcoR1+Eag1/ OX1.9 blot an intense signal was detected in the $2.8 \mathrm{~Kb}$ unmethylated band and very little signal in the $5.2 \mathrm{~Kb}$ band which in normal females results from the inactivated $X$ chromosome. In addition an extremely faint band in the full mutation size range was also detected. Relative faintness of the $5.2 \mathrm{~Kb}$ band in females is a warning that much of the signal may be spread in the full mutation size range, possibly difficult to detect on an EcoR1+Eag1/ OX1.9 blot. However a Bgl11/OX1.9 failed to detect a condensed and easily visible upper band. Hence the relative faintness of the $5.2 \mathrm{~Kb}$ band and of the full mutation band could not be explained by somatic heterogeneity of the FMR-1 expansion allele or by skewed X inactivation.. On resampling the patient, cytogenetic analysis revealed the patient to be $X O / X X$ mosaic with less than $10 \%$ of cells being $X X$. The results reported here illustrate the need for a careful inspection of the EcoR1+Eag1/ OX1.9 blot. A faint 'smear' on such a blot would usually be clearer on a Bgl11/OX1.9 blot. On the few occasions when it is not, alternative explanation should be sought and investigated.

\subsection{7}

An atypical case of Prader-Willi displaying abnormal methylation patterns with normal biparental inheritance Carpenter, Karen 1; Sharif, A L 1; Young, I D 2; Heath, $M^{3}$ Curnock, D 4; Cross, G S 1

Departments of 'Molecular Genetics, 2 Clinical Genetics, 3 Cytogenetics and 4 Paediatrics, Nottingham City Hospital, Nottingham NG5 1PB

Around $60 \%$ of Prader-Willi patients are shown to have a deletion in the paternal copy of $15 q 11-13$. Maternal uniparental disomy (UPD) accounts for almost all of the remaining cases. A 1 year old male was referred as a possible Prader-Willi case. The complete spectrum of symptoms usually associated with this disease were not apparent; in particular the facial features were atypical. High resolution cytogenetic analysis of the patient showed a normal karyotype. FISH analysis using the SNRPN probe did not show a $15 q$ deletion. DNA methylation studies were performed using the probe PW71B. Southern blot hybridisation of both Hind III/Hpall and Hind III/Hha I digests showed that the bands corresponding to the paternal copy of 15q11-13 were greatly reduced in intensity compared to the maternal copy. Similar results were obtained using probe KB17 with Not V/ Xba I DNA digests. CA repeat polymorphism analysis was also performed. The markers D15S11, GABRAB3, D15S113, D15S97 and GABRA5 were found to be informative and showed that the patient had normal biparental inheritance. These results suggest the presence of either a microdeletion which has occurred de-novo in the Prader-Willi critical region or an inherited mutation leading to a disruption in the normal imprinting mechanism.
13.008

Towards the fully automated identification, mutation analysis and routine diagnosis of Human Disease Genes Henfrey, Richard 1

PE Applied Biosystems (A Division of Perkin-Elmer), 850 Lincoln Centre Drive, Foster City, CA 94404, USA

The Human Genome Project has now come of age, and attention is switching to the clinical application of the mapping and sequence information obtained from within the genome, with particular interest being shown in the identification of genes associated with clinically important conditions, such as cancers, diabetes, hypertension, asthma, and schizophrenia. Automation of techniques such as high throughput sequencing and linkage mapping using fluorescence-labelled microsatellite markers have allowed the rapid location of genes associated with monogenic and multifactorial disease, and combined with the increasing availability of well-characterised families for genetic studies, the link between the clinician and molecular geneticists is being dramatically shortened. Developments in technology are now being focused on offering improved tools for the routine screening and diagnosis of human diseases (for example cystic fibrosis, Fragile- $X$, myotonic dystrophy, Duchenne Muscular Dystrophy), paternity testing, preimplantation diagnosis and organ typing. In addition to high throughput applications, emphasis is also being placed on the total automation of PCR based assays. The latest developments in multi-colour fluorescence technology for genetic analysis will be presented using applications data relevant to the clinical environment, such as the TaqMan Assay for mutation analysis, OLA in CF testing and HLA typing by DNA sequencing.

13.009

Cystic Fibrosis mutation detection by modified PCR restriction enzyme digest

Palmer, Sheila

Institute of Medical Genetics, University Hospital of Wales, Heath Park, Cardiff, CF4 4XN

The Cystic Fibrosis mutations $1898+1 \mathrm{G}>\mathrm{T}$ and $\mathrm{R} 117 \mathrm{H}$ represent $5 \%$ and $0.5 \%$ of Welsh CF mutations respectively. Neither of these mutations create or destroy a restriction site, therefore detection has previously been by dot blot hybridisation with radiolabelled allele specific oligonucleotides or sequencing. Primers adjacent to the above mutations were designed with a base substitution towards the 3 ' end which in combination with the mutation create or destroy a restriction enzyme site. For $1898+1 G>A$ the modified primer 5'TAAGTAAGGTATTCAAAGAAGAT3' is used as the reverse primer with the Zielenski 12i5' primer as the forwards primer. In this case the underlined $G$ replaces a $C$ resulting in the creation of an EcoRV site in the presence of the mutation. In the case of the R117H modified primer a $G$ replaces an A which creates a Cfol site in the normal sequence; this is lost in the presence of the mutation. In this case the forwards primer which is modified: 5'CCGGATAACAAGGAGGAGC3' and the reverse primer is Zielenski 4i3'. We expect to use this technique for more mutations and if appropriate determine whether certain types or positions of substitution are more successful than others. 